\title{
Haemophilia: a rare cause of bleeding after ear surgery Varghese $L^{1}$, Ahmed $S^{2}$, Michael $R C^{3}$, Rupa $V^{4}$
}

\begin{abstract}
:
Introduction: Excessive bleeding during ear surgery is a troublesome problem which could be related to inadequate infiltration, active infection, trauma to the dura or jugular bulb or sudden hypertension. When the problem recurs in the postoperative period, despite intraoperative control of the field, a bleeding diathesis should be suspected. Case report: We present two adult patients in whom excessive intraoperative and postoperative bleeding associated with ear surgery led to the diagnosis of congenital mild haemophilia A. Conclusion: This case report highlights the fact that excessive perioperative bleeding for which no other cause can be found in an adult patient should suggest the presence of a bleeding diathesis like haemophilia A or B for which detailed coagulation workup is required. Any subsequent surgery would need to be performed with administration of specific perioperative medication to reduce the possibility of excessive bleeding.
\end{abstract}

Keywords: Perioperative haemorrhage; congenital haemophilia A

Bangladesh Journal of Medical Science Vol. 15 No. 01 January'16. Page : 135-138

\section{Introduction:}

Haemophilia is an inherited X-linked bleeding disorder, typically seen in young males. It is classified into haemophilia $\mathrm{A}$ and $\mathrm{B}$, based on deficient coagulation factor VIII and IX respectively ${ }^{1}$. Haemophilia $\mathrm{A}$ is more commonly encountered with an incidence of about one in 5000 male births ${ }^{2}$. The disease generally affects males on the maternal side and a family history of bleeding is commonly obtained. However almost one-third of these patients may not have a family history as both Factor VIII and Factor IX genes are prone to spontaneous mutation ${ }^{3}$. Bleeding usually occurs from deep tissues rather than from surface tissues. Although many bleeding diathesis may be first diagnosed at surgery, it is extremely rare for a diagnosis of congenital haemophilia A to be made in a previously undiagnosed adult patient. A literature search using Medline, Google scholar and Scopus did not show any previous reports of haemophilia A diagnosed postoperatively after any surgery. We report two rare cases of congenital hemophilia A in adult patients whose diagnosis was established after elective ear surgery to highlight this rare but treatable entity.

\section{Case Report:}

Case 1: A 37 year old gentleman from West Bengal, diagnosed with left sided mucosal chronic suppurative otitis media, underwent transcanal tympanoplasty by underlay technique using temporalis fascia graft harvested from the left supraaural region. Intraoperative bleeding was moderate but controllable. The immediate postoperative period was uneventful without any sign of a collection or soakage of the dressing. On the third postoperative day, he presented with a tender, fluctuant swelling over the surgical site associated with oedema and ecchymosis around the left eye. About $3 \mathrm{ml}$ of serosanguinous fluid was drained from the wound site and a pressure dressing using a mastoid bandage was applied. Presuming that the collection was due to secondary infection, he was prescribed a course of oral cloxacillin. On the seventh postoperative day, a collection was noted at the same site and $10 \mathrm{ml}$ of blood drained. Since bleeding from the wound site persisted, emergency

1. Lalee Varghese

2. Saud Ahmed

3. Rajiv C Michael

4. V. Rupa

Department of Otorhinolaryngology, Christian Medical College, Vellore, India.

Corresponds to: Dr Lalee Varghese, Department of Otorhinolaryngology, Christian Medical College, Vellore, Tamil Nadu, India. PIN: 632004. E mail: laleevarghese@yahoo.co.in 
Intrinsic pathway

Extrinsic pathway

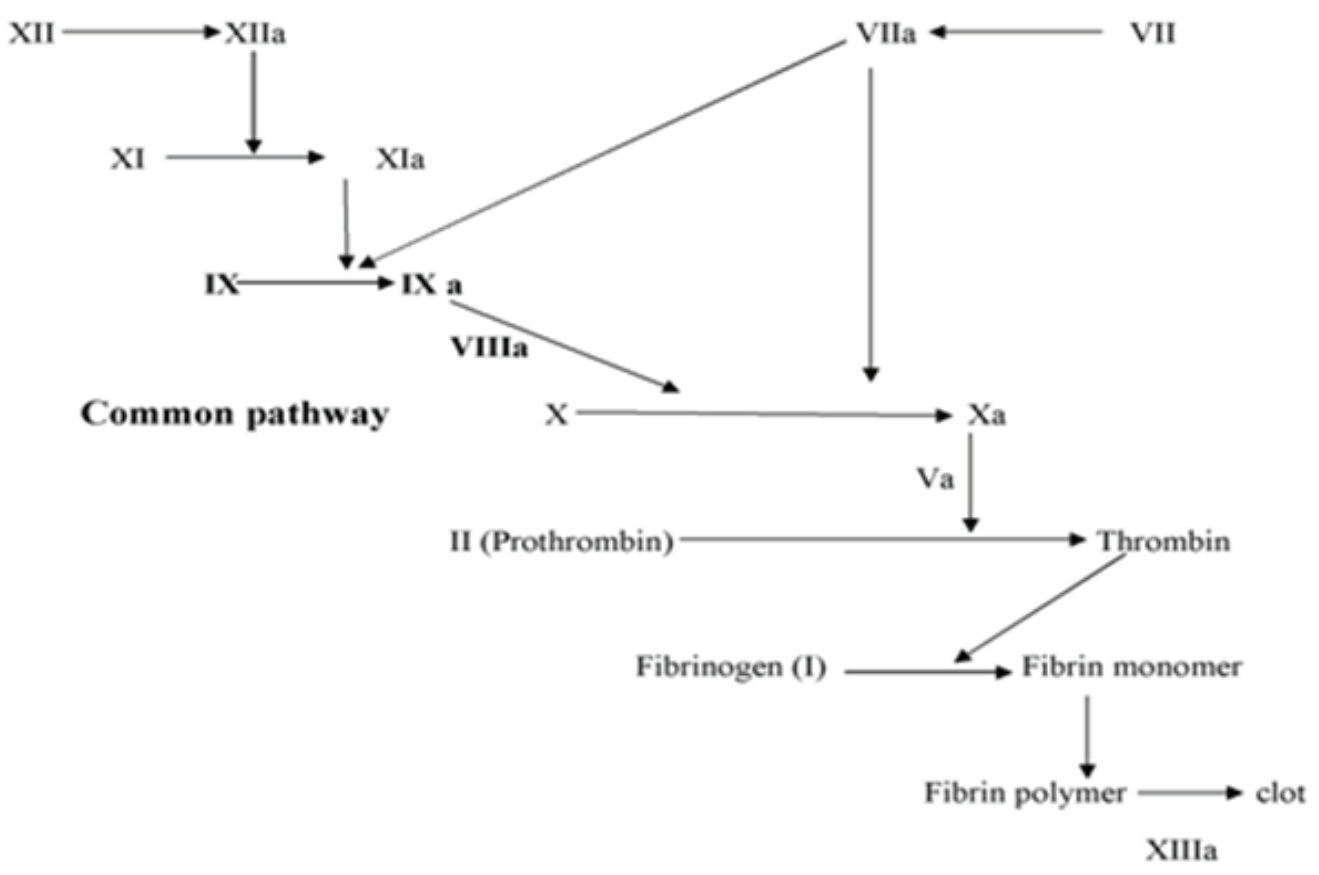

Figure:1 Coagulation pathway showing site of action of factor VIII and factor IX which are deficient in haemophilia A and B respectively

exploration of the wound under local anaesthesia was performed and a haematoma evacuated. There was diffuse bleeding from the wound which was cauterised and haemostasis achieved. Subsequent coagulation work up revealed a platelet count of 120000 per cubic millimetre, prothrombin time of 11.4 seconds (control 10-12.5 seconds) and activated partial thromboplastin time (APTT) of 51.7 seconds (control 25.1-36.7 seconds). In view of deranged APTT, an opinion was sought from the haematologists and a complete coagulation work up performed. The abnormalities noted were a prolonged APTT (which was corrected with control plasma) and a factor VIII level of $18.1 \%$. On the basis of these results, he was diagnosed to have mild haemophilia A. Since there was no further bleeding, no active intervention was recommended by the haematologist and he was kept on follow up. At review after four months, the tympanic membrane graft had taken up well and the wound had healed completely.

Case 2: A 35 year old male with bilateral otosclerosis causing bilateral, symmetrical conductive hearing loss was taken up for left stapedotomy under local anaesthesia. The procedure had to be abandoned due to excessive bleeding and lack of cooperation by the patient. Presuming that the bleeding was due to the patient's inability to cooperate for local anaesthesia, re-exploration was scheduled under general anaesthesia two days later. At surgery, the bleeding was again found to be excessive, despite normal blood pressures. The wound from the previous surgery was still raw and the accompanying diffuse bleeding was presumed to be due to the lack of efficacy of the infiltration used. A decision was made on the table to perform right stapedotomy instead, using a new bottle of 1\% xylocaine with 1: 1000 adrenaline infiltration. Surgical consent was taken from the patient's relatives. The surgery went off uneventfully. On the eighth postoperative day the patient presented with bleeding from the right ear following a bout of cough. The patient was taken up for re-exploration on an emergency basis. A bleeding point which was identified at the lower end of the incision site was cauterised and haemostasis achieved. Subsequent haematological evaluation revealed that while his platelet count and prothrombin time were normal, APTT was prolonged (patient 45.8 seconds; control 25.1-36.7seconds) which corrected in 
mixing studies. Factor IX, XI and XII levels were normal, but the factor VIII level was only $24.5 \%$, establishing a diagnosis of mild haemophilia A.

\section{Discussion:}

Haematological disorders are usually identified in patients when they are subject to minor trauma or undergo dental extraction. Bleeding following otological surgery is a very rare occurrence and an unusual manner of presentation of a bleeding diathesis. Common causes for excessive intraoperative bleeding during ear surgery include bleeding from the incision site due to inadequate infiltration by decongestant or inadequate maintenance of haemostasis by electrocautery/ ligation, dural trauma, injury to the sigmoid sinus or jugular bulb, active middle ear infection with granulations or hypertension. If any of these situations are not managed adequately, the patient can present later with a wound collection or haematoma. A less frequently encountered etiology is a hitherto undiagnosed bleeding diathesis. Patients with mild bleeding disorders can also present similar problems and, hence, these disorders should be considered once all the other common causes are excluded.

Haemostasis after surgery is a finely balanced process where blood vessel wall injury by surgical intervention initiates the coagulation pathways (Figure: 1) that control bleeding.

Any aberration in this process can lead to a bleeding disorder which can be classified as coagulation factor deficiencies, platelet disorders, vascular disorders or fibrinolytic defects ${ }^{4}$. In hemophilia A platelet count, bleeding time and prothrombin time are normal but clotting time and APTT are prolonged. The severity of bleeding depends on the residual factor concentration and based on this haemophilia can be classified into severe with factor level $<0.01 \mathrm{IU} / \mathrm{ml}(<1 \%$ of normal), moderate with factor level 0.01 to $0.05 \mathrm{IU} / \mathrm{ml}$ (1 to $5 \%$ of normal) and mild with factor level $>0.05$ to 0.40 $\mathrm{IU} / \mathrm{ml}$ (more than 5 to $40 \%$ of normal) ${ }^{5}$.

Haemophilia A is classically caused by a congenital deficiency of factor VIII, but an acquired form due to inhibitors to factor VIII has also been described in adult patients in the literature. The diagnosis of acquired haemophilia $\mathrm{A}$ is based on the demonstration of an isolated prolongation of the activated partial thromboplastin time (APTT), not corrected by incubating the patient's plasma with equal volumes of normal plasma (mixing study), associated with reduced factor VIII levels and evidence of FVIII inhibitor activity ${ }^{6}$. In both our patients APTT corrected in mixing studies thus confirming the diagnosis of congenital haemophilia. Typically, patients with moderate or severe congenital haemophilia A are diagnosed early in life because the bleeds occur even with minor trauma and it is the mild cases (similar to the 2 cases described) that are missed. In the first patient described, the recurrent large haematoma at the postoperative site prompted us to suspect an underlying bleeding disorder. In the second patient, abnormal intra and postoperative bleeding from both ears called for a detailed coagulation work up. On enquiry, both patients did not give any history of bleeding tendencies in the past. There was also no family history of bleeding or bleeding tendency. For haemophilia A, factor VIII concentrates are the treatment of choice ${ }^{7}$. Pharmacological agents that are useful in the management of haemophilia include desmopressin, tranexamic acid and epsilon aminocaproic acid. Desmopressin, a synthetic analogue of vasopressin that boosts plasma levels of FVIII and IX is useful both for treatment of patients with mild or moderate haemophilia A and prevention of bleeding in carriers of haemophilia ${ }^{8}$. Tranexamic acid is an antifibrinolytic agent that competitively inhibits the activation of plasminogen to plasmin, promotes clot stability and is useful as adjunctive therapy in haemophilia. Epsilon aminocaproic acid (EACA) is similar to tranexamic acid, but is less widely used as it has a shorter plasma half-life, is less potent, and is more toxic. In the postoperative period, drugs that affect platelet function, particularly acetylsalicylic acid and nonsteroidal anti-inflammatory drugs (except certain COX-2 inhibitors) should be avoided. Paracetamol is a safe alternative for analgesia ${ }^{3}$.

As both the patients described had minor deep bleeding and factor VIII levels were only marginally deranged, transfusion or infusion of factor concentrates was not required. It is possible that administration of tranexamic acid in the perioperative period might have reduced the chances of bleeding in these patients, had these patients been diagnosed earlier. Certainly, if any other elective or emergency surgery is performed in such patients in future, the surgeon is forewarned and better prepared to deal with possible intraoperative or postoperative bleeding.

\section{Conclusion:}

This case report highlights the fact that a patient who has excessive intraoperative or postoperative 
bleeding for which no other common cause is detected, should be suspected of having a bleeding diathesis like haemophilia A or B and be evaluated accordingly. Any subsequent surgery in patients diagnosed with mild haemophilia would need to be performed with administration of pre or perioperative medication to reduce the possibility of excessive bleeding during this period.

\section{Acknowledgements:}

The authors would like to acknowledge with gratitude Dr Biju George, Professor of Haematology, Christian Medical College, Vellore, for his valuable input into the haematology perspective.

\section{References:}

1. Israels S, Schwetz N, Boyar R, McNicol A. Bleeding disorders: Characterization, dental considerations and managements. J Can Dent Assoc 2006;72:827

2. Mannucci PM, TuddenhamEG.The hemophilias - from royal genes to gene therapy. $\quad N$ Engl $J$ Med 2001;344(23):1773-9. http://dx.doi.org/10.1056/NEJM200106073442307

3. Srivastava A, Brewer AK, MauserBunschotenEPet al. Guidelines for the management of hemophilia.Haemophilia2013;19:e1-47. http://dx.doi.org/10.1111/j.1365-2516.2012.02909.x

4. Patton LL. Bleeding and clotting disorders. In: Burket's oral medicine: diagnosis and treatment. 10th ed. Hamilton (ON): BC Decker;2003. p.454-77.

5. White GC, 2nd, Rosendaal F, Aledort LM, et al. Definitions in hemophilia. Recommendation of the scientific subcommittee on factor VIII and factor IX of the scientific and standardization committee of the International Society on Thrombosis and Haemostasis. Thromb Haemost 2001;85:560.

6. Franchini $M$, et al. Laboratory, clinical and therapeutic aspects of acquired haemophilia A. Clin Chim Acta 2008;395:14-18. http://dx.doi.org/10.1016/j.cca.2008.05.003

7. Stanworth SJ. The evidence-based use of FFP and cryoprecipitate for abnormalities of coagulation tests and clinical coagulopathy. Hematology Am SocHematolEduc Program 2007;00: 179-86. http://dx.doi.org/10.1182/asheducation-2007.1.179

8. Mannucci PM. Desmopressin (DDAVP) in the treatment of bleeding disorders: the first 20 years. Blood 1997;90:2515-21. 